\title{
COMMISSION 20: POSITIONS AND MOTIONS OF MINOR PLANETS, COMETS AND SATELLITES
}

\author{
PRESIDENT: D. K. YEOMANS \\ VICE-PRESIDENT: H. RICKMAN \\ Secretary: E. L. G. Bowell \\ Organizing Committee: K. Aksnes, J. -E. Arlot, E. L. G. Bowell, A. Carusi H. Rickman, V. A. Shor, D. \\ K. Yeomans, L. H. Wasserman, J. -X. Zhang
}

\section{I. Introduction (D. K. Yeomans)}

In the past triennium, the output of the Commission continued its explosive growth within a rather broad range of activities. In comparison with the previous triennium (which also saw impressive growth), the current period witnessed a $26 \%$ increase in the number of numbered minor planets (7041 minor planets), a $46 \%$ increase in the number of minor planet orbits computed (14 154) and a $187 \%$ increase in the number of optical Minor Planet observations (260 616). In addition, there were 65 radar Doppler and time delay measurements made during the triennium bringing the total to 269 observations of 40 minor planets and 5 comets.

The past triennium has been marked by new discoveries and circumstances that have helped re-define solar system modelling activities. Some 34 new trans-Neptunian objects were discovered to add to the two discovered during the previous triennium and these discoveries have established the reality of the Kuiper belt (or Edgeworth-Kuiper belt) of objects at, or beyond, the distance of Pluto. In fact, for these objects that are in a 2:3 libration with Neptune, the discoverers have suggested the unofficial name Plutinos. The number of Centaurs on unstable orbits in the realm of the outer planets doubled to six. Comet Shoemaker-Levy 9 split into nearly two dozen fragments during a July 1992 close Jupiter approach and, two years later, each of the fragments collided with Jupiter. As well as providing a challenge to dynamicists to represent this extraordinary phenomena, the event energized the planetary science community into re-thinking the nature of the cometary nucleus itself. Mention should also be made of the two puzzling objects, 1996 N2 and 1996 PW. Comet Elst-Pizarro (P/1996 N2) is active but with an orbit that looks very much like that of a main belt asteroid while asteroid $1996 \mathrm{PW}$ is inactive yet has a comet-like orbit that is very nearly parabolic. Clearly the dividing line between comets and asteroids is no longer clearly drawn.

The observations of the natural satellites continued unabated with the focus of activity on the Jovian and Saturnian systems - perhaps because of the Galileo and Cassini spacecraft missions that are, or soon will be, observing these fascinating bodies in situ. However, the triennium was unusually quiet with respect to occultation events and there is nothing significant to report in this area.

Some 123 new Near-Earth Objects (NEOs) were discovered during the triennium bringing the total to 378. Largely through the efforts of A. Carusi, The Spaceguard Foundation was successfully established in an effort to promote and coordinate activities for the discovery, pursuit, and orbital calculations of NEOs on an international level.

The world wide web has provided rapid access to several data sources mentioned in the following sections including the Minor Planet Center, the Long-Term Evolution of Short Period Comets (A. Carusi), as well as a natural satellite astrometric database at the Bureau des Longitudes. In this regard, mention should also be made of the Lowell Observatory's database for minor planet orbits (http://www.lowell.edu).

In the following reports, there are numerous references to a few journals. These frequently mentioned journals and their abbreviations include Earth, Moon, and Planets (EM\&P), Celestial Mechanics and Dynamical Astronomy (CM), and Astronomy and Astrophysics ( $A B A)$. 
It is a sad duty to record the deaths of M.P. Candy, Bruno L. Morando, N. Samojlava-Yakhontova and Jean-Louis Sangier. With them, the Commission has lost some of its most active and esteemed members.

\section{Minor Planets (B. G. Marsden)}

\subsection{GENERAL}

Continuing the long-standing Commission 20 arrangement, the Minor Planet Center (MPC) at the Smithsonian Astrophysical Observatory in Cambridge, Mass., and the Minor Planets and Comets section of the Institute for Theoretical Astronomy (ITA) in St. Petersburg have again collaborated on organizing the collection of astrometric observations and the computation of orbital elements and ephemerides. The results are principally disseminated via the monthly Minor Planet Circulars (MPCs) and annual Efemeridy Malykh Planet (EMP). The MPC work is carried out by director B. G. Marsden and associate director G. V. Williams, with contributions from S. Nakano in his capacity as Liaison in Japan, as well as from C. M. Bardwell and D. W. E. Green. The ITA work is carried out by chief Yu. V. Batrakov and deputy chief V. A. Shor, with contributions from Yu. A. Chernetenko, I. A. Filippova, G. R. Kastel', O. M. Kochetova, N. K. Sumzina, O. O. Vasil'kova and T. A. Vinogradova.

During the triennium July 1993-June 1996 a total of 5072 pages of $M P C$ s were issued. Although this number is smaller than the 5802 of the previous triennium, it is important to note that the use of $\mathrm{T}_{\mathrm{EX}}$ for printing the $M P C$ s from 1993 Sept. 1 onward means that the 1993-1996 output corresponds to some 9500 old-style pages. The number of pages in the EMP increased from 552 in the 1994 edition to 627 in the 1996 edition. Observations and orbits from the $M P C$ s are also available electronically in the Extended Computer Service, while the EMP is also available as $S T A M P$, a diskette version. ITA also issues the more general software package CERES for the computation of orbits and ephemerides.

Another important change in MPC operations in Sept. 1993 was the introduction of the series of Minor Planet Electronic Circulars (MPECs). As noted in the report of Commission 6, the principal motivation for this was to simplify the operation of the Central Bureau for Astronomical Telegrams and to reduce the number of IAU Circulars that are issued. The MPECs, 541 of which had been issued by the end of June 1996, are used to provide rapid information about unusual minor planets, including both near-Earth objects and Kuiper Belt candidates. More detailed information and more frequent orbital updates can therefore be provided than was possible in the past. The computations for and the mainly automatic preparation, verification and dissemination of an $M P E C$ can often be completed in less than 10 minutes. The $M P E C$ s have also proven useful for providing follow-up information for comets (although the original announcement and first orbit computation continue to be provided by means of IAU Circulars). Furthermore, special monthly MPECs are used to show the current observational status of the unusual objects (including comets and Kuiper Belt candidates) and the critical list.

The complete tape of observations issued in Aug. 1995 contained 546043 observations of numbered minor planets and 377542 of unnumbered minor planets. The combined total is increasing at a rate of 14 percent per year and surpassed one million in March 1996. While almost all of the increase comes from current observations currently reported, some 10 percent is due to the extraction by Williams of nineteenth-century measurements from the literature and his re-reduction of these data on the system of the PPM. He has also utilized the Digital Sky Survey to remove quite a few erroneous observations from the files that are demonstrably linear groupings of stars, single stars, edge-on galaxies and the like.

At the end of June 1996 there were 7041 numbered minor planets, a 26-percent increase during the triennium. As suggested in the previous report, it was decided to issue the Catalogue of High-Precision Orbits of (Unnumbered) Minor Planets on an annual basis. By arrangement with ITA, the opportunity was taken in the 1996 edition to include the 518 newly-numbered objects that would not be added to the EMP until 1997. This Catalogue also contained 5183 orbits for unnumbered multiple-opposition objects and 269 perturbed orbits for single-opposition objects. The late-1994 edition of the Catalogue of Orbits of (Single-Opposition) Unnumbered Minor Planets referred to 15587 objects, a number that had increased to 17514 by mid-1996. Allowing also for the increase in multiple-opposition orbits since the start of the year, the total number of minor-planet orbits then amounted to 30730 .

The MPC can be accessed on the World Wide Web at http://cfa-www.harvard.edu/cfa/ps-/mpc.html. Some low-precision orbital information, as well as statistical information on unusual orbits and on discovery information about minor planets generally, is automatically maintained there. "The NEO Confirmation Page", set up in March 1996, has already proven very useful, in that it provides information 
about suspected near-Earth objects, generally recorded on only one night, in need of confirmation. In addition, "The $1996 \mathrm{JA}_{1}$ Page" allowed users to compute perturbed, topocentric ephemerides - an invaluable aid for observers of this record large Earth-approacher that attained apparent magnitude 11 but had a parallax of 50 arcmin.

A second edition of the Dictionary of Minor Planet Names was published by L. D. Schmadel. Explanations are provided for all but 124 of the names assigned to 4253 of the first 5655 numbered minor planets.

Following the earlier success with (951) Gaspra, in situ observations were made of (243) Ida by the Jupiter-bound Galileo space probe in August 1993. Some of the Galileo images indicated that the object has a satellite, named Dactyl, the reality of which could subsequently be supported by rather stringent dynamical constraints.

\subsection{OBSERVATIONS AND ORBITS}

A total of 260616 optical astrometric observations made of minor planets during the triennium had been published in the Minor Planet Circulars or included in the Minor Planet Center's accessible files by early Aug. 1996. This was 87 percent larger than the total made during the previous triennium. Some 24 percent of the observations referred to the minor planets that had by then received permanent numbers. About 28 percent of the numbered objects were not observed at all during the triennium, while 31 percent were observed ten or more times. Thirty-three numbered minor planets were observed at least 100 times and six more than 200 times. The best observed objects were (1620) Geographos (398 observations) and (6489) Golevka (364 observations), which passed by the Earth at distances of $0.033 \mathrm{AU}$ in Aug. 1994 and $0.034 \mathrm{AU}$ in June 1995, respectively.

Radar observations were also made of a number of minor planets, notably by S. Ostro using the radio telescopes at Arecibo and Goldstone, and these have been utilized in orbital computations by D. K. Yeomans and P. W. Chodas at the Jet Propulsion Laboratory. In June 1995, echoes were successfully detected at Evpatoria and Kashima of transmissions from Goldstone reflected from (6489) Golevka-this object having in fact been named in honor of this international experiment, following a suggestion by $A$. L. Zajtsev of the Evpatoria site.

Provisional designations were given to 22274 initially unidentified minor planets, 71 percent more than during the preceding triennium. The number would be much greater if the former practice of allowing single-night detections were still followed.

The 260616 observations were obtained at 183 different observatories in 29 different countries. Ninetysix of the observatories made more than 100 observations and 31 more than 1000 . Just five countries-the United States, Chile, Japan, Italy and the Czech Republic-contributed 85 percent of the observations, the U.S. fraction alone being 48 percent (at 38 different observatories, including the Hubble Space Telescope). Here, "Chile" means almost exclusively the European Southern Observatory and, specifically, programs by Belgian astronomers. Some 70 percent of the observatories (including all but two of the 46 Japanese observatories and all of the 19 Italian observatories) are operated by amateur astronomers. Nevertheless, amateurs contributed only 20 percent of the observations, and only one of the 12 leading observatories is an amateur installation. Those 12 observatories, which produced 77 percent of the observations, are as follows:

(1) Spacewatch, 84868 observations by T. Gehrels, J. V. Scotti, D. L. Rabinowitz, R. Jedicke and J. Montani with the scanning-CCD camera on the Steward Observatory's 0.9-m reflector at Kitt Peak. Designed mainly to search for NEOs, this enormous program also yields several thousand single-night detections of unknown objects each month.

(2) European Southern Observatory, 44097 observations, mainly measured by E. W. Elst from photographic exposures with the 1-m Schmidt.

(3) Oak Ridge, 10373 observations by R. E. McCrosky with the 1.5-m Wyeth reflector. This is still the principal program designed for follow-up rather than discovery, and about one-third of the minor planets currently being numbered are numbered solely because of observations made at Oak Ridge at their latest oppositions.

(4) Oizumi, 9842 observations made single-handedly by the amateur astronomer T. Kobayashi using a $0.25-\mathrm{m}$ reflector.

(5) Kleť, 8566 observations by J. Tichá, M. Tichý and Z. Moravec, mainly using a 0.57-m reflector. 
(6) Palomar, 8532 observations, mainly from the two NEO photographic programs run by E. M. Shoemaker and E. F. Helin with the $0.46-\mathrm{m}$ Schmidt. These programs came to a halt around the end of 1994.

(7) Xinglong, 6777 observations, made under the direction of J. Zhu using a $0.60-\mathrm{m} \mathrm{Schmidt.} \mathrm{This}$ program started in April 1995.

(8) NEAT, 6601 observations, carried out in a collaboration between the Jet Propulsion Laboratory and the U.S. Air Force with the 1-m GEODSS telescope at Haleakala. This NEO program, under the direction of E. Helin, had its first successful run in December 1995.

(9) Observatoire de la Côte d'Azur, 6356 observations with the 0.9-m Schmidt at Caussols, mainly by Elst and C. Pollas photographically, the measurements being by Elst.

(10) Siding Spring, 6017 observations from plates taken with the 1.2-m U.K. Schmidt and CCD images with a $1.0-\mathrm{m}$ reflector. The observations are by R. H. McNaught, G. J. Garradd, D. Asher and D. Steel, and there is a concentration on NEOs.

(11) AMOS, 4174 observations, a NEO-concentration follow-up program with the 1.2-m reflector at the Air Force Maui Optical Station at Haleakala, partly in association with E. Helin at JPL. Measurements are by J. Africano, P. Kervin, P. Sydney and others, and the first results were obtained in March 1995.

(12) La Palma, 3320 observations, mainly of numbered minor planets with the Carlsberg Meridian Circle, under the direction of L. V. Morrison.

Orbital elements were computed for a total of 14154 minor planets, 46 percent more than during the previous triennium. The number of orbits computed and published was actually considerably more, for the count refers only to the most recent results for each object, and in addition, "Väisälä" orbits (representative computations on the assumption that an object is at perihelion or aphelion) are automatically computed for all objects observed at least twice. Of the 14154 orbits, some 53 percent involved the linkage of observations at multiple oppositions or long-arc single oppositions that made it necessary to consider planetary perturbations. Twenty different orbit computers were involved in the work, although seven of them were responsible for 97 percent of the computations, their individual percentages being Williams 55, Nakano 15, E. Bowell 12, Marsden 10, E. Goffin 2, Bardwell 2 and Kobayashi 2. Most of the multiple-opposition orbits involve the establishment of identifications. In addition to the above, significant contributions to this identification work were made by K. Ichikawa, K. Kinoshita and T. Urata.

The triennium produced the discoveries of 123 new near-Earth asteroids, bringing the total to 378 . Since these objects are defined to be those with perihelion distances as high as 1.3 AU, and since some of those found by Spacewatch are too small to do significant damage even if they did hit the Earth, it is useful to define a subset of "potentially hazardous asteroids" (PHAs), which have absolute magnitude $\leq 22.0$ and whose orbits currently come within $0.05 \mathrm{AU}$ of that of the Earth. If one is interested only in possible collisions within the next couple of centuries, it is generally sufficient to simplify this minimum-distance computation and take the Earth's orbit as circular. According to this definition there are $91 \mathrm{PHAs}$, of which 27 were discovered during the triennium 1993-1996 and 20 during the preceding triennium; 44 of the PHAs are single-opposition objects, and 24 of these have observed arcs of less than 30 days. Among the recent short-arc PHAs are $1995 \mathrm{CR}$ and $1996 \mathrm{JA}_{1}$, which made the closest known PHA approaches to the Sun $(0.12 \mathrm{AU})$ and $\operatorname{Earth}(0.003 \mathrm{AU})$, respectively. The latter object was discovered by T. B. Spahr and C. W. Hergenrother during their photographic patrol near Tucson for minor planets at high ecliptic latitudes. There is some evidence that the cessation of the Palomar photographic programs caused a noticeable reduction in discoveries that persisted for the whole of 1995 . If so, recovery was apparent in 1996, as programs like NEAT began to make up for the loss.

In the last report the discovery of two transneptunian objects by D. Jewitt and J. Luu at Mauna Kea was a special point of interest. During the 1993-1996 triennium no fewer than 34 more transneptunian objects were discovered, again mainly at Mauna Kea. Particularly interesting was the realization that perhaps $30-40$ percent of these objects appear to have orbits like that of Pluto, approaching and even crossing Neptune's orbit at perihelion but prevented from encountering Neptune because of a 2:3 meanmotion resonance. Since only about half of the objects have been observed at more than one opposition, most of the orbit solutions are completely uncertain, but it also appears that two objects, $1995 \mathrm{DA}_{2}$ and $1994 \mathrm{JS}$, may be involved with the 3:4 and 3:5 resonances. Since all these new discoveries, presumably members of the Kuiper Belt (membership in which should therefore also be prescribed for Pluto itself), are in the magnitude range 22-24, follow-up is difficult-but essential if anything quantitative is to be learned about this population. 
Three new centaurs, objects with unstable orbits in the realm of the giant planets, were also discovered, thereby doubling the number of known objects in this class. Two of them, $1994 \mathrm{TA}$ and $1995 \mathrm{DW}_{2}$ were found during the Mauna Kea Kuiper Belt survey, while $1995 \mathrm{GO}$ was found by Spacewatch. The perihelion and aphelion distances of $1995 \mathrm{DW}_{2}$ are 18.9 and $31.2 \mathrm{AU}$, respectively.

On the theoretical front, two important new methods of preliminary orbit determination address the multiple solutions that arise when one considers that complete revolutions might occur between pairs of observations. A method by R. H. Gooding (RAE Tech. Rpt. 93004, 1993; submitted to CM, 1996) is based on his elegant and complete solution of the Lambert problem; practical applications to the orbits of minor planets have been discussed by Marsden and Gooding (BAAS 26, 1021, 1994). L. K. Kristensen (Astron. Nachr. 316, 261, 1995) has devised a four-observation method that makes use of the fact that an unperturbed orbit is confined to a plane. K. Muinonen and E. Bowell (Icarus 104, 255, 1993) have utilized Bayesian principles to predict orbital uncertainties; although their principal applications are to minor planets that have been widely observed at several oppositions, they have made a start, with L. H. Wasserman (Planet. Space Sci. 42, 307, 1994), on the more troublesome matter of handling single-opposition orbits.

\section{Comets (H. Rickman)}

\subsection{NEW DESIGNATION SYSTEM}

At the General Assembly in 1994, the commission decided to approve a new designation system for comets, whereby both the letters and the Roman numerals following the year are abolished and replaced by a combination of one capital letter and one Arabic numeral. The year is now uniquely the year of discovery, the letter indicates the half-month of discovery (identical to the system used for minor planets), and the numeral gives the order of discovery in that half-month. When an orbit is computed, a prefix is added with the usual meanings of "P/" for periodic comets (orbital period $<200 \mathrm{yr}$ ) and "C/" for comets that are not periodic. If a comet is designated but no orbit can be computed, the prefix is "X/". The prefix " $\mathrm{D} /$ " is given to comets whose returns can no more be predicted for whatever reason, be it physical disappearance or a number of missed returns, after which the ephemerides are hopelessly inaccurate. Finally, an object designated as a comet which later turns out to be an asteroid by physical appearance gets the prefix "A/".

These designations are preliminary as far as the prefixes are concerned, since practically all imaginable transitions may really occur. But in addition there is a definitive numbering sequence for periodic comets, somewhat analogous to the one in use for minor planets. When a periodic comet is confirmed, usually by being recovered at a following apparition or observed to pass aphelion, it is assigned a number in chronological sequence. This numbering applies also to objects originally numbered as minor planets, if these have shown unmistakable cometary activity, the cases in question so far being (2060) Chiron = 95P/Chiron and (4015) Wilson-Harrington $=107 \mathrm{P} /$ Wilson-Harrington. Some numbered comets of the past are now obviously defunct and have thus acquired the prefix "D/", e.g., 3D/Biela and 5D/Brorsen. For comets that are established as periodic and under more or less continuous observation, having been missed only occasionally, the recoveries are routine. These are no longer given designations. On the other hand, designations are given to recoveries of one-apparition comets or comets with relatively large ephemeris uncertainties such as the long-lost ones of the "D/" kind or comets with long orbital periods.

The new system is effective from the start of 1995 , but the numbering sequence naturally applies to all periodic comets by backward extrapolation according to the dates when the periodicities were established. Likewise, new-style designations are available for all cometary apparitions before 1995 . They are listed with translation tables to the old-style designations in the 1995 edition of the Catalogue of Cometary Orbits by Marsden and Williams. Marsden (63.103.021) gives a historical background and description of the new designation system.

Guidelines for the naming of comets have been under discussion within the bodies set up at the 1994 General Assembly, but a resolution of this issue has to await the General Assembly in 1997.

\subsection{DISCOVERIES AND RECOVERIES}

During the past triennium several search programmes were closed, that had previously produced a large fraction of all comet discoveries, and as a result the number of discoveries has dropped considerably. 
While the triennium 1990-93 saw 23 long-period (LP) and 20 short-period (SP) comet discoveries, the one of 1993-96 only produced 16 LP and 11 SP comet discoveries. The latter include two rediscoveries of long-lost comets, namely, 113P/Spitaler identified as comet $1993 \mathrm{U} 2$ by J. Scotti during the Spacewatch survey, and $122 \mathrm{P} /$ de Vico rediscovered visually by several Japanese amateurs as a 7 th magnitude comet designated $1995 \mathrm{~S} 1$.

It is worth noting that the amateurs have.increased their discovery rate so that the past triennium counts 13 amateur discoveries against only 14 professional. In reality, the distinction between the two categories is becoming less meaningful than it used to be, since many of the current amateur discoveries were made at well-equipped observatories especially in Japan, where the work is carried out with great efficiency, aided by the experience gained at professional sites.

Of the 16 LP comets discovered, seven were in the "professional" category - all of them using photographic techniques with Schmidt telescopes. The magnitude range of these discoveries was $~ 13-17$. Of the nine amateur discoveries of LP comets, six were visual (magnitudes $~ 6-11$ ), two were photographic at magnitudes $\sim 10-12$, and one [comet C/1994 G1 Takamizawa-Levy)] was shared between the two modes. The 11 SP comet discoveries had a certain preference (seven/four) for the professional category, though much smaller than that of the preceding triennium (17/3). Three of these (including the rediscovery of $113 \mathrm{P} /$ Spitaler) were made using a CCD detector by the Spacewatch team, so this technique, although still suffering from its small field of view, is nonetheless beginning to have a significant impact on the SP comet discovery rate. The continued presence of visual discoveries of SP comets by amateurs is noteworthy - during the past triennium, not only was $122 \mathrm{P} /$ de Vico rediscovered in this manner, but there was also P/1994 P1 (Machholz 2) that was seen at 10th magnitude. This comet was noted to have split before discovery, and the splitting may have increased its brightness. In fact, sporadic shifts of activity level seem commonplace among SP comets of small perihelion distances, and this together with the often difficult geometric conditions is likely to create a niche for continued visual discoveries.

During the past triennium the most prolific discovery sites were Palomar and Siding Spring Observatories with five cases each. The main worry for the future is the upcoming closure of the Siding Spring program, because this currently represents nearly all discoveries from the southern hemisphere. There is already a serious imbalance of the latitudinal discovery distribution with a 21/6 overweight for the northern hemisphere during the past three years. A delayed discovery of a LP comet at Siding Spring occurred in 1993, concerning comet C/1978 G2 (McNaught-Tritton), as R. H. McNaught reported a further 1978 image of this comet from the old plates and B. G. Marsden established its orbit, by assuming identity with another image from 1979, so that yet another image was found on a plate from 1980 (IAUC 5966).

In addition to the discoveries, important recoveries were made for 11 SP comets that had previously only been observed at their discovery apparitions (in 1985-1991). This led to assigning permanent numbers to these comets, and thus there were in total 125 permanently numbered comets at the end of the triennium. The critical recovery observations were done with the Spacewatch telescope (by J. Scotti and colleagues) in 10 of the 11 cases, including one (114P/Wiseman-Skiff) which was shared with the MMT telescope. The remaining recovery observations (of $124 \mathrm{P} / \mathrm{Mrkos}$ ) were done independently by Hergenrother and the New Mexico amateur W. Offutt. All 11 recoveries were made using the CCD technique.

An additional recovery of special importance, though of the standard kind since the comet was already numbered, was that of $46 \mathrm{P} /$ Wirtanen in June 1995 by $\mathrm{H}$. Böhnhardt et al. This comet is the current target of the Rosetta spacecraft mission and was approaching the Sun for its last perihelion passage before the instrumentation and observing strategy had to be frozen. The recovery was remarkable since it was made at a remote orbital position (4.6 AU from the Sun) in a crowded star field near the galactic center direction, and the image was exceedingly faint. Before the end of the triennium, the comet had already developed substantial activity though its magnitude was still no brighter than 20 .

Last but not least, the triennium 1993-96 saw the visual discoveries, both by amateurs, of two LP comets that would become exceptionally bright. The first of these, C/1995 O1 (Hale-Bopp), has not yet come to perihelion but was remarkably bright (10th magnitude) for its heliocentric distance of more than $7 \mathrm{AU}$. Its initially CO-driven activity was taken over by $\mathrm{H}_{2} \mathrm{O}$ before the end of the triennium, whereby the brightening slowed down, but the first naked-eye sightings were reported in June 1996, nine months before perihelion. The second comet, C/1996 B2 (Hyakutake), was discovered at 11th magnitude but rapidly approached the Earth on its way to perihelion and thus presented an impressive display to northern observers during the last week of March 1996. 


\subsection{ASTROMETRY AND ORBITS}

Observations of remote comets continued to be pursued. While these are often made explicitly for the benefit of physical understanding of the nuclei, e.g., to measure the photometric cross-sections without coma contamination, they are also of great use for getting precise knowledge of the orbits, including nongravitational effects, and thus improving the accuracy of the ephemerides. The past triennium saw the numbering of one periodic comet (117P/Helin-Roman-Alu 1) due to aphelion observations. Observers particularly active in remote detections were $\mathrm{K}$. Meech and $\mathrm{O}$. Hainaut $(B A A S 27,1123)$, and reports on special programmes for observing extremely remote comets were given by Hainaut and Meech (BAAS 27, 1125) and Hainaut et al. (62.103.007). The latter programme also involved the last observation of $1 \mathrm{P} /$ Halley (IAUC 5935) at magnitude $\mathrm{V}=26.5$ and heliocentric distance $18.8 \mathrm{AU}$ in Jan. 1994, using the ESO 3.5-m NTT.

Astrometric observations were routinely published in the MPCs, and the number during the past triennium was 18747, more than twice the number during 1990-93. No single comet is responsible for this increase, the best observed ones being D/1993 F2 (Shoemaker-Levy 9) with 2801 observations of individual nuclei, C/1995 O1 (Hale-Bopp) with 1204 observations, C/1996 B2 (Hyakutake) with 841, 29P/Schwassmann-Wachmann 1 with $698,19 \mathrm{P} /$ Borrelly with 529, and C/1993 Q1 (Mueller) with 525 . Only the single best observed comet of the preceding triennium (109P/Swift-Tuttle with 718 observations) would enter into this list, showing that a general increase in astrometric coverage has occurred. The most prolific sites were Spacewatch (2111 observations), Kuma Kogen (1838), Chiyoda (1462), Klet (1302) and Marl (1030). Again, only one observatory of the preceding triennium (Oizumi; 1088 observations) could have competed with these. The 2 nd, $3 \mathrm{rd}$ and 5 th on this list of five are amateur observatories with small-field instruments. The geographic distribution of the astrometric observations is even in longitude (36\% in Japan, $25 \%$ in the US, $24 \%$ in the four most productive European countries) but strongly biassed toward the northern hemisphere. Moreover, Svoren (58.103.017) summarized the results of photographic astrometry at Skalnaté Pleso in 1986, and Protitch-Benishek and Olević (63.103.014) described astrometry of $1 \mathrm{P} /$ Halley and $21 \mathrm{P} /$ Giacobini-Zinner in 1985-86.

The MPCs provided the principal forum for continual publication of orbit determinations, mainly for newly discovered or recovered comets, and tabulations of orbital elements for all cometary apparitions were given in the yearly editions of the Catalogue of Cometary Orbits by B. G. Marsden and G. V. Williams. The 11th edition of 1996 contains orbits for 1470 apparitions of 883 comets, whereof 185 of short period (124 of these having been observed at more than one perihelion passage). 'Original' and 'future' orbits were given for 291 comets of long period. The Comet Handbook, edited by S. Nakano and D. W. E. Green, was published yearly by the $I C Q$ with orbits and ephemerides for upcoming apparitions. Muinonen $(M N 280,1235)$ examined the covariances of orbital elements as determined for comet C/1995 $\mathrm{O} 1$ (Hale-Bopp), showing that the primary uncertainty occurs along one eigenvector in element space.

Yeomans (61.102.028) reviewed recent advances in understanding the nature of cometary nongravitational forces. The nongravitational effects of $46 \mathrm{P} /$ Wirtanen and their interpretation were reviewed by Jorda and Rickman (63.103.071). Sekanina (58.102.018) especially considered the component normal to the orbital plane as caused by outgassing from discrete active regions and showed that the data for comet $71 \mathrm{P} /$ Clark allow conclusions on its nuclear properties. He also investigated the discontinuities in nongravitational effects exhibited by some comets and concluded (58.103.008) that these can be explained by the sudden turn-on of new discrete sources.

Models of the force that are asymmetric with respect to perihelion passage were considered by several authors. Sitarski (61.102.041) used a time-shifted model with parameters $A_{1}, A_{2}, A_{3}$ and force function $\mathrm{g}[\mathrm{r}(\mathrm{t}-\tau)]$ to link all the apparitions of comet $22 \mathrm{P} / \mathrm{Kopff}$ and later found (53.103.018) that this gave an excellent fit to the data of the 1994 apparition. Linkages using a model based on force amplitude A and orientation angles $\eta, I, \varphi$ for a discrete outgassing source at latitude $\varphi$ were made by Sitarski for 45P/Honda-Mrkos-Pajdušáková (Acta 45, 763) and 51P/Harrington (Acta 46, 29), by Sitarski and Todorovic-Juchniewicz for $14 \mathrm{P} /$ Wolf $(61.103 .152)$ and by Sitarski and Królikowska for $46 \mathrm{P} /$ Wirtanen $(A \& A 310,992)$. Szutowicz and Rickman (62.103.051) attempted a further improvement and extension of this model, using an outgassing function based on the visual light curves, for comet $6 \mathrm{P} / \mathrm{d}$ 'Arrest. Bolatto et al. (63.102.022) used the time-shifted model for LP comets in order to establish the perturbation on orbital energy to be expected from nongravitational effects.

The identity of comet D/1827 M1 Pons-Gambart with the oriental comet of 1110 was claimed by Hasegawa and Nakano (63.103.028; PASJ 47, 699), with the latter paper also arguing for the identity of 
comets $\mathrm{C} / 1861 \mathrm{~J} 1$ and $\mathrm{C} / 1337 \mathrm{M} 1$ with the comets of 1500 and 1468 , respectively. In 1995 , comet images were reported from old Lowell Observatory plates taken by C. W. Tombaugh in Jan. and Sep. 1931. The latter object, comet $\mathrm{D} / 1931 \mathrm{R}$, was suggested identical to comet $84 \mathrm{P} /$ Giclas by $\mathrm{R}$. J. Bouma (IAUC 6168), and B. G. Marsden succeeded in linking the objects. Sitarski (Acta 46, 47) refined the linkage, deducing also nongravitational parameters for the comet.

\subsection{DYNAMICS, POPULATIONS, AND ORIGINS}

A general review was presented by Festou et al. (58.102.022), and reviews focussing on cometary origins were given by Bailey $(E M \& P 72,57)$ and Marov $(62.102 .004)$, on cometary dynamics by Fernández (61.102.027), and on populations by Kresák (61.102.025), Shoemaker et al. (63.103.002) and Luu (61.102.024), the latter specializing in the Edgeworth-Kuiper belt.

The action of Galactic tides on the Oort cloud was studied by Matese et al. (EMEP 72, 7; Icarus $116,255)$ who found that different distributions of dark disk matter yield different amplitudes of timemodulation of the cometary influx rate. Dybczyński and Prestka $(62.102 .034 ; E M E P$ 72, 13) also studied the influx mechanism into observable orbits from the cloud, using numerical integrations. Giant molecular cloud encounters were examined by Marochnik et al. (58.102.049) who concluded that spiral density waves including bar-like structures could be excited, though only temporarily. Stellar passages through the inner Oort cloud leave signatures that are not consistent with the present orbital distribution of LP comets according to Weissman $(58.102 .031 ; E M E P ~ 72,25)$. The impulse approximation for computing easily the effects of such encounters was revisited by Dybczyński (CM 58, 139), and Petrovskaya (EM\&P 72, 31) computed effects of stellar encounters based on analytic theory.

Sharma and Khanna (61.102.008) and Tomanov et al. (62.102.001) studied the rate of capture of interstellar comets by the solar system and their resulting orbital distributions. The issue of the absence, hitherto, of detections of any clearly interstellar comets was treated by Sen and Rana (58.102.013) who concluded that the present nondetection is consistent with current theories of the Oort cloud and solar system formation, while Weissman $(61.102 .033 ; B A A S \mathbf{2 7}, 1123)$ instead invoked an effect earlier unaccounted for, i.e., that the interstellar ejection and Oort cloud storage by Uranus and Neptune is very inefficient. Neslušan $(A \& B A 306,981)$ analyzed the perihelion directions of LP comets, accounting for the north-south asymmetry of comet discoveries, and concluded like many earlier authors that the directions are randomly distributed.

The problem of explaining the distribution of inverse semimajor axes of LP comets, and in particular the peak height of the new Oort cloud comets, was discussed by Whipple (61.102.047) who concluded that there may be no evidence for a present cometary shower. Emel'yanenko and Bailey $(E M E P$ 72, 35) found that there is a problem posed by the very rapid fading involved and, especially for perihelia beyond 1.5 AU, the observed distribution indicates renewal of old comets. They thus concluded that dormancy characterizes most LP comets with large perihelion distances. Salitis (EMEP 72, 41) considered the effect of stellar perturbations on the orbital distribution of LP comets. Bailey et al. (MN 281, 916) made a detailed study of the orbital evolution of C/1995 O1 (Hale-Bopp), analyzing different possible end states, and concluded that the comet may be considered dynamically young.

The capture of comets into short-period orbits was studied by Valtonen and Zheng (58.102.001) who found the inclination bias for capture perturbations upon close encounters to be strong, and by Zheng et al. (EM\&P 72, 45) who claimed good agreement with the orbital distributions of Jupiter family and Halley-type comets, simulating capture from an isotropic Oort cloud including all four giant planets. Valtonen et al. (EMEP 71, 219) studied captures from the Oort cloud into Earth-crossing orbits and concluded that terrestrial craters of at least $20 \mathrm{~km}$ diameter could be formed mostly by impacts of such comets. But Fernández and Gallardo $(A \& A$ 281, 911) found that when Jupiter alone governs the capture process, a randomly oriented source of LP comets cannot yield an orbital distribution of SP comets in agreement with the one observed. Marzari et al. ( $A \& S A$ 299, 267) argued that a certain fraction of the SP comets might be escaped collisional fragments from the Trojan clouds. Quite different ideas were discussed by Radzievskij (61.102.039; 63.102.018).

Previous work on cometary capture had sometimes made use of increased planetary masses, and Valsecchi and Manara (58.102.007) demonstrated the alteration of the orbital energy perturbation distribution caused by this. Zheng (62.102.127) constructed a trivariate perturbation distribution for parabolic comets encountering a planet in a circular orbit by computing, separately for different values of the en- 
ergy perturbation, a distribution of inclination perturbations leading directly to the perturbations of perihelion distance via the Tisserand criterion.

Short-period comets being subject to a long sequence of close encounters with Jupiter, their dynamics is known to be strongly chaotic. Tancredi $(A \& A 299,288)$ thus computed Lyapunov times of around 50-100 yr for Jupiter family comets, and Nakamura and Yoshikawa (PASS 43, 801) found the round-trip error for such comets to approach unity within 1000-2000 yr. The special effect of nongravitational forces under such conditions was investigated by Emel'yanenko (58.103.036), finding that they can be neglected only over an interval spanning one or two encounters with Jupiter.

The collisions of comet D/1993 F2 (Shoemaker-Levy 9), in the following SL-9, with Jupiter stimulated interest in planetary collision probabilities. Neslušan (58.102.006) and Steel (58.102.047) both estimated terrestrial impact probabilities for comets in general, and Nakamura and Yoshikawa (Icarus 116, 113) did likewise for Jupiter and its satellites. Obrubov (EMEP 72, 293) concentrated on $1 \mathrm{P} /$ Halley and 96P/Machholz 1 and their collision probabilities with Jupiter and the terrestrial planets, and Lohinger et al. (EMEP 71, 225) specialized on orbital variants of $1 \mathrm{P} /$ Halley. The orbital evolution of SL-9 itself was subject to many studies. Benner and McKinnon (Icarus 118, 155) and Chodas and Yeomans (The Collision of Comet Shoemaker-Levy 9 and Jupiter, Cambridge Univ. Press, 1996, pp. 1-30) found that the satellite capture had been more long-lasting than any previously known one, involving regular oscillations of eccentricity and inclination due to secular solar perturbations, and the latter (also BAAS 27, 1202) found statistical evidence for a capture in the late 1920's. Meanwhile, Sitarski (63.103.100) from a different analysis found SL-9 to have been captured directly preceding the 1992 close approach. Kary and Dones (Icarus 121, 207; BAAS 27, 1112) studied the statistics of temporary satellite captures in general and thus the expected frequency of SL-9 like events. A search for comets in Jupiter's vicinity by Tancredi and Lindgren (61.102.019) gave no positive result.

A new edition of Long-Term Evolution of Short-Period Comets by A. Carusi et al. became available on the WWW (http://titan.ias.fra.cnr.it/), and an upcoming new edition of the Catalogue of Short-Period Comets was announced by Medvedev et al. (EMEP 71, 215). Studies of orbital evolutions specializing in individual SP comets were published by Benest and Gonczi $(58.103 .034 ; 58.103 .063 ; 61.103 .048)$ for comets in resonance with Jupiter. The case of P/1994 P1 (Machholz 2) was studied by Asher and Steel $(M N 280,1201)$ in relation to possibly related meteor displays. Nakamura and Yoshikawa (58.103.035) integrated the motions of several objects including 95P/Chiron and 29P/Schwassmann-Wachmann 1 over $10^{5} \mathrm{yr}$ backward and forward.

The motion of 109P/Swift-Tuttle from $703 \mathrm{BC}$ to $\mathrm{AD} 2392$ was studied by Yau et al. (61.103.140), placing emphasis on the visibility conditions and the lack of a need to include nongravitational effects even over an interval of more than $2000 \mathrm{yr}$. An update on the orbital motion from 1737 to 1992 was given by Marsden et al. (58.103.156), including a suggestion of the identity, later confirmed, with the ancient comets of +188 and -68 . A more extended integration over $40000 \mathrm{yr}$ centered on the present was made by Chambers (63.103.037).

Valsecchi et al. (Icarus 118, 169) studied the dynamics of objects in orbits like that of $2 \mathrm{P} /$ Encke and concluded that paths exist linking such objects both to the main asteroid belt and to the Jupiter family of comets. A genetic relationship between the bodies of the so-called Taurid complex was not found to be supported. Steel and Asher ( $M N$ 280, 806) looked into mechanisms to explain the orbital dispersion of this complex assuming a common, recent origin and found that nongravitational forces offer a reasonable possibility. A relationship with the Hephaistos group was also found possible, and in a separate study (62.103.087) Steel and Asher concluded that comet D/1766 G1 (Helfenzrieder) may indeed be a member of the latter group. Orbital integrations by Kurahashi and Nakamura (63.102.009) indicated that within $30000 \mathrm{yr}$ some transfer into Apollo-Amor asteroid orbits is possible. But a very small probability of such transfer emerged from long-term integrations by Harris and Bailey (IAJ 23, 151).

Levison and Duncan (61.102.020) made integrations over $10^{7}$ yr for the known SP comets, finding that a limit at Tisserand parameter $=2$ is seldom crossed and thus forms a good separating line between the Jupiter family and Halley-type comets. Important end states were found to be hyperbolic ejection $(92 \%)$ and sungrazing orbits $(6 \%)$. Detailed results with plots of the individual evolutions were given in the SwRI Catalog of Short-Period Comet Orbit Integrations by H. Levison and M. Duncan. Long-term orbital evolutions of Halley-type comets were computed by Bailey and Emel'yanenko $(M N 278,1087)$ who found evidence for important secular resonant effects acting over $10^{5}-10^{6} \mathrm{yr}$. A survey of all the major dynamical features influencing the long-term evolution of Halley-type comets was given by Chambers 
( $\mathrm{PhD}$ thesis, Univ. of Manchester).

The evolution of Jupiter family comets was simulated by Tancredi (61.102.045) including fading and dormancy periods. The issue of major splitting events contributing to the Jupiter family was reviewed by Bailey et al. (63.103.003) and Pittich and Rickman (61.102.001) investigated the time scale for relaxation of the orbit distribution after such an event. Different evidence for cometary aging, based on Levison and Duncan's long-term integrations, was reviewed by Whipple (EMEP 72, 69), while Markovich and Markovich (58.102.002) found that SP comets keep their orbital distribution fixed but fade in magnitude over their observed intervals. The issue of the existence of a large population of small comets, with sub$\mathrm{km}$ sized nuclei, was revisited by Brandt et al. (EMEP 72, 243), arguing for a search programme to characterize this population.

\section{Satellites (J. -E. Arlot)}

\subsection{ASTROMETRIC OBSERVATIONS}

\subsubsection{Observations of the Satellites of Mars:}

During this triennium, all the observations of Phobos and Deimos (1963 - 1988) made by the staff members of Goloseevo Observatory (Ukraine) were processed using the PPM catalog. About 2000 improved positions of the satellites and Mars have been obtained. These results are in preparation for publication. At the USNO, photographic observations were continued by D. Pascu with the 26-inch refractor at Washington D.C. However, due to a shortage of $103 \mathrm{aG}$ plates from Kodak, a hiatus in these photographic observations may occur for the satellites of Mars as well as for the satellites of Jupiter and Saturn.

\subsubsection{Observations of the Galilean Satellites of Jupiter:}

Direct Observation of Positions: Nikolaev Observatory (Ukraine): During the period from January 25,1994 to June $15,1994,33$ photographic positions of the satellites were obtained with the zonal astrograph $(\mathrm{D}=154 \mathrm{~mm}, \mathrm{~F}=2042 \mathrm{~mm})$. These observations are included in the paper by $\mathrm{G}$. $\mathrm{K}$. Gorel' and L. A. Gudkova "Photographic observations of the satellites of Jupiter and Saturn in 1991-1994 (Ninth Report)". This paper contains 193 positions of satellites, and while it is not yet published, it is available by application to the authors. During the period from May to July 1995, 16 absolute positions of the satellites with respect to the FK5 were determined by photoelectric meridian observations using their meridian circle equipped with CCD-microscopes. Pulkovo Observatory: Astrometric observations of the Galilean satellites made with the 26-inch refractor in 1976-1981 have been completed. The jovicentric positions and mutual distances of the satellites have been obtained. The residual differences, computed by comparison with Arlot's theory, were used to investigate the occasional and systematic observational errors and to estimate the accuracy of the theory. The internal accuracy of the observations is estimated to be 0.03 " with the external errors being equal to 0.05 "- 0.17 " depending on the distances between satellites. The longitude correction of J IV was determined and the improvement of Jupiter's positions by the measurement of satellites positions with respect to fundamental stars was investigated (Kisseleva, Proceedings of IAU Symposium 172, S. Ferraz-Mello, B. Morando and J. E. Arlot eds, Kluwer Academic Press, 1996). U.S. Naval Observatory (Washington D.C.): The extensive set of photographic observations continued. These data are used to update the ephemerides for JPL's Galileo project and they are also used at the Bureau des Longitudes for ephemeris development.

Photometric Observations: Mutual events of the Galilean satellites observed in 1991 were reduced and catalogues of positions are in press (Kaas et al. in Astron. J., and Arlot et al. in $A B A$ ). Eclipses of the Galilean satellites were observed during the period 1990-1991 by Westfall and published in $A L P O J, 37$, $154,1994$.

\subsubsection{Observations of the Faint Satellites of Jupiter:}

The results of observations of J VI, J VII, and J VIII made in 1987-1989 at Goloseevo Observatory (Ukraine), Kitab (Uzbekistan), and Majdanak (Uzbekistan) by the staff members of Goloseevo Observatory have been published (Izhakevich et al., Kinematika i Physica Nebesnykh Tel vol.10, n1, Kiev, 1994). As a result of the Uppsala-ESO survey of asteroids and comets, Hernius et al.(A\&A Supp. Ser. 115, 295, 1996) published photographic observations of JVI-XIII taken at ESO, Chile and at AAO, Siding Spring, Australia. Shelus et al. (Astron. J. 106, 2573, 1993) published photographic observations of the faint outer 
satellites of Jupiter made at the McDonald Observatory; Whipple et al. (Astron. J. 112, 316, 1996) published their observations of J VI-XIII made during the 1994 and 1995 oppositions. CCD observations of J $\mathrm{V}$ and J XIV were made using the 61-inch astrometric reflector of the U.S. Naval Observatory at Flagstaff.

\subsubsection{Observations of the Satellites of Saturn:}

Direct Observation of Positions: U.S. Naval Observatory: Satellites I-VIII are continuously observed photographically with the 26-inch refractor in Washington, D.C. while CCD observations of satellites XII, XIII and XIV were made at Flagstaff with the 61-inch astrometric reflector (Rhode and Pascu, BAAS 25, 1235, 1993 and $B A A S$ 26, 1024, 1994). Pulkovo Observatory: Saturn's satellites I-VI and VIII were observed photographically with the 26 -inch refractor. Some 46 plates were taken with 6 or 7 exposures on every plate during the period from August to November 1995. The planetocentric positions and mutual distances of the satellites were measured with an internal precision on the order of 0.1 " and an external precision of 0.1 " -0.2 ". A paper on the results of the observations has been prepared. Satellites S I - S VI were also observed during the period from August to October, 1995 with a CCD camera installed on the 26-inch refractor. As a result, about 100 mutual positions of the Satellites have been obtained with an equivalent internal precision of 0.05 " -0.10 " and an external precision of 0.1 "-0.2." Four mutual events were also observed, but with relatively low precision; results of these observations have been prepared for publication. Engelhardt Observatory: Satellites S IV, S V, and S VI were observed with the astrograph $(\mathrm{D}=400 \mathrm{~mm}, \mathrm{~F}=3780 \mathrm{~mm}$ ) during the period from September 1994 to February 1995 (19 plates) and from November 1995 to April 1996 ( 7 plates). The plates have been processed. High Altitude Station of Engelhardt Observatory in Zelenchuk: Observations of S III, S IV, S V, S VI, and S VIII were observed with the Zeiss astrograph $(D=400 \mathrm{~mm}, F=2000 \mathrm{~mm})$ in September 1994 (29 plates) and in December 1994 (11 plates). As a result of a joint project between Queen Mary and Westfield College (QMWC) and the Royal Greenwich Observatory, La Palma CCD observations at the oppositions in 1990,1991,1993 and 1994 were published for Saturn's satellites $(A \& A 269,564-567,1993 ; A \& A$, in press). At nearly every opposition from 1968 up to 1995, Y. Hatanaka of the Japanese National Astronomical Observatory (NAO) made photographic astrometric observations of 7 satellites of Saturn with the Zeiss 26-inch refractor $(\mathrm{f}=10 \mathrm{~m})$. Although observations made under constant conditions for such a long period of time are valuable, only a small portion of the observations have been published. (Publ. National Astron. Obs. Japan, vol.4, No.2, 23-65, 1996). Using the opportunity of the ring plane crossing, observations were made of Saturn's faint inner satellites using the Hubble Space Telescope (HST), infrared arrays at Palomar Observatory and CCD's at Pic du Midi Observatory, France and at the Observatorio Nacional-LNA in Brazil.

Photometric Observations: During the ring plane crossing of 1995 , extensive observing campaigns were undertaken of the eclipses by Saturn and of the mutual events. Within the Former Soviet Union, considerable work was undertaken to organize the community for the mutual events in Saturn's satellite system during 1995-1996. These efforts were undertaken by the Celestial Mechanics Department of Sternberg Institute (GAISH), Moscow, headed by N.V. Emel'yanov. At the Sternberg Institute (GAISH) South Station in Crimea, two events were observed photoelectrically with the Zeiss-600 - the occultation of Dione by Rhea on August 6,1995 and the eclipse-occultation of Enceladus by Tethys on September 14, 1995. At the Astrophysical Institute (Kazakhstan Republic) observations of events were made with 2 instruments. The RZ-1000 $(\mathrm{D}=1000 \mathrm{~mm})$ was used at Assy station at an altitude of $2750 \mathrm{~m}$ and the RZ-600 $(D=600 \mathrm{~mm})$ was used at Almaty (Alma-Ata) at an altitude $1450 \mathrm{~m}$. Altogether, fifteen events were observed. Recording of the events was carried out with a CCD-matrix, with 82 to 547 separate frames being taken during every event. A light curve for the eclipse-occultation of Enceladus by Tethys on September 14, 1995 was obtained with the CCD-matrix. In addition to the above observations, several attempts to observe events in Saturn's satellite system were undertaken in Alma-Ata by visiting astronomers of GAISH, Engelhardt Observatory and Pulkovo Observatory. Altogether, eleven mutual events were observed with a photoelectric photometer using the regime of photon counting but, unfortunately, the results of all these observations have a low quality due to unmodelled variations of scattered light intensity; they hardly can be used for improving the satellite theories. The Bureau des Longitudes organized an observing campaign resulting in 20 observations of eclipses and 94 observations of mutual events. Due to the difficulties of the reduction, only a portion of these observations will be useful. 


\subsubsection{Observations of the Satellites of Uranus:}

D.H.P. Jones and J.-E. Arlot are reducing La Palma CCD observations of the Uranian satellites made at the oppositions in 1990 and 1991. These are being analyzed using a numerical integration. CCD observations were made by Veiga and Viera-Martins ( $A \& A$ Supp. Ser. 107, 551, 1994). The reduction of these data and of older photographic data were made using the motion of Uranus. In addition, they published data from photographic plates (A\&A Supp. Ser. 107, 559, 1994) and from CCD observations ( $A \& A$ Supp. Ser. 113, 557, 1995). Pascu, Rhode and Seidelmann made CCD observations of Miranda with the 61-inch astrometric reflector at Flagstaff. HST astrometric observations of the inner satellites discovered by Voyager were reduced by Pascu et al. (BAAS 186, 1302, 1995).

\subsubsection{Observations of the Satellites of Neptune:}

Positions of these satellites were published by Veiga et al. (A\&A Supp. Ser. 115, 319, 1996) and Nereid was observed by Pascu, Rhode and Seidelmann at Flagstaff.

\subsubsection{Miscellaneous:}

A method for observing faint satellites near bright planets was given by Veiga and Viera-Martins (A\&A Supp. Ser. 111, 387, 1995). The Working Group on Natural Planetary Satellites has continued to gather observations in order to build a database. This database is resident on a computer at the Bureau des Longitudes and is reachable by Internet at http://www.bdl.fr/. The Galilean satellite data are on-line and other data are available via the ftp server at ftp.bdl.fr/pub/NSDC.

\subsection{THEORETICAL WORKS AND COMPARISONS TO OBSERVATIONS}

\subsubsection{Miscellaneous:}

J. Lieske $(C M 57,473,1993)$ published an algorithm for the IAU north poles and rotation parameters of the planetary satellites. Kinoshita $(C M 57,359,1993)$ studied the motion of the orbital plane of a satellite due to a secular change of the obliquity of its central planet.

\subsubsection{Satellites of Mars:}

R. Jacobson (JPL) made a fit of the Morley/Sinclair theory to all the available astrometric observations from 1877 to 1988 as well as the Mariner 9, Viking and Phobos spacecraft observations (AAS Paper 96-140, AS/AIAA Space Flight Mechanics Meeting, Austin, Texas, 1996). At the 25th Lunar and Planetary Science Conference, Smith et al. published masses of Mars, Phobos and Deimos from the analysis of Mariner 9 and Viking Orbiter tracking data.

\subsubsection{Galilean Satellites of Jupiter}

The Sampson-Lieske theory of the motion of the Galilean satellites was fit to mutual events observations, and constants were published by Vasundhara et al. (Proceedings of the IAU Symposium 172, Ferraz-Mello, Morando and Arlot eds, Kluwer Academic Press, 1996) and new ephemerides built. In addition, Lieske (JPL Eng. Mem. 314-545, 1994 and JPL Eng. Mem. 312-583, 1995) fit the theory and built ephemerides.

\subsubsection{Other Jovian Satellites}

Solovaya et al. (Planetary and Space Science 42, 8, 685, 1994) investigated the orbital motion of J VIII. Chapront and Rocher analyzed observations and published ephemerides under a new representation of the faint outer satellites of Jupiter JVI-JXIII ( $A \& A$ in press).

\subsubsection{Satellites of Saturn:}

The Saturn satellite pair Titan - Hyperion was studied by Kirsanov of the ITA. A rough numerical orbit for Hyperion was used as a reference in Encke's method of numerical integration. The accuracy of such an integration was considerably higher than the straightforward one. Some 473 observations of Titan and 93 observations of Hyperion (from 1967 to 1981) were considered in order to improve the satellites' orbital elements. The mean $\mathrm{O}-\mathrm{C}$ value for Hyperion is 0.47 ." The normality of the distributions of $\mathrm{O}-\mathrm{C}$ residual values along and across the visible orbits of the satellites was studied. Harper and Taylor (QMWC and RGO) compared the quality of observations of the Saturn's satellites made during the period 1874 to 1988 resulting from a fit of all data to the analytical theories ( $A \& A$ 284, 619, 1994). Pre- 
dictions of the eclipses by Saturn and mutual events were made by Arlot and Thuillot (Icarus 105, 427, 1993) and ephemerides using Dourneau's theory were provided to observers. The staff members of the Sternberg Institute (GAISH), Moscow, under the leadership of N.V.Emel'yanov have developed an integrated software package (MONS-EPH) for ephemerides in support of satellite observations (Emel'yanov, Pis'ma $v$ Azh. 22, 153, 1996). Precise modern theories are realized in the package for the majority of the 59 natural satellites in the solar system. For the remainder, there are no precise analytical theories so that approximate ones with sufficient accuracy for ephemeris calculations are provided. The package is intended not only for calculation of positions of satellites for any moment of time but also for the calculation of circumstances of observations (positions of primary, the Sun and the Moon and its phase, etc.). The package also aids the search for mutual events in the satellite systems and provides calculations of their circumstances. All these results are displayed graphically and in numerical form. The package is distributed among a number of observatories of the world. Using the package, the circumstances of all mutual events in Saturn's system in 1995-1996 were computed and published by Emel'yanov et al. and Emel'yanov (Astron. Zh. 71, 794, 1994: Astron. Zh. 72, 604, 1995). Mutual events among Galilean satellites in 1997-1998 have also been calculated and the corresponding ephemerides are in press. Jacobson provided a re-determination of the minor satellite orbits (numerically integrated and precessing ellipse) from the original Voyager observations, astrometric observations through 1994, and 1995 Hubble Space Telescope (HST) observations (BAAS 27, No.3, 1202-1203, 1995). Jacobson fit a numerically integrated Phoebe orbit to astrometric observations from 1904 to 1994 and the Voyager 2 imaging observations. He also provided numerically integrated orbits for the eight major satellites using astrometric observations from 1971 to 1995 , Voyager imaging observations, 1980 mutual event observations, and 1995 HST observations (Meeting of the AAS Division on Dynamical Astronomy, US Naval Observatory, Washington, D.C., 1996). Vienne and Duriez $(A \& A 297,588,1995)$ fit their own theory (TASS) to observations and made a comparison with Earth based observations. Message (CM 56, 277, 1993) discussed the second order long-period motion of Hyperion.

\subsubsection{Satellites of Pluto}

Bursa and Milan (EMEP 65, 3, 291, 1994) studied the effect of the tidal and rotational distortion on the figures of Pluto and Charon. A search for small satellites of Pluto was made by Flynn et al. (BAAS 27, 1340, 1995) using the HST archives. Studying the mutual events light curves, Young and Binzel (Icarus 108, 219, 1994) made a new determination of radii and limb parameters for Pluto and Charon. Stern et al. (Icarus 108, 234, 1994) explored the possibility of satellites in the inner Pluto-Charon system by studying the dynamical and observational constraints.

\subsection{RINGS}

\subsubsection{Rings of Saturn}

Spahn et al. (CM 57, 391, 1993) studied the gravitational influence of the satellite Pan on the radial distribution of ring-particles in the region of the Encke division in Saturn's ring. A. Bosh analyzed several occultations of the Saturnian system observed from ground-based observatories, from Voyager 1 and 2 and from HST. This work was part of her $1994 \mathrm{Ph}$. D. Thesis at the Massachusetts Institute of Technology entitled "Stellar occultation studies of Saturn's rings with the Hubble Space Telescope."

\subsubsection{Rings of Uranus and Neptune}

Photometry of the rings was made by Currie et al. (BAAS 26, 1376, 1994) from HST observations. French and Nicholson (BAAS 27, 857, 1995) studied the edge waves and librations in the epsilon ring. The origin of the rings of Uranus and Neptune was studied by Colwell and Esposito (JGR, Planets 98, 7387, 1993). Hanninen and Porco (BAAS 26, 1418, 1994) studied the stability of the arcs of Neptune. Ferrari and Brahic made a systematic analysis and quantitative photometric study of Voyager 2 images of the Neptunian narrow rings (Icarus 111, 193, 1994). Colwell (Planetary and Space Science 42, 1139, 1994) studied the hypothesis of the disruption of small planetary satellites creating new narrow dusty rings around the planet Jupiter, Saturn, Uranus and Neptune. 


\section{Working Group on Near-Earth Objects (A. Carusi)}

The second triennium of activity of the Working Group on Near-Earth Objects (WGNEO) has been dense with events and quite fruitful. In the following a brief account of the major achievements will be provided, together with suggestions for the future.

\subsection{THE VULCANO WORKSHOP}

The Vulcano Workshop "Beginning the Spaceguard Survey" (September 1995) was organized by the WGNEO in response to a precise statement contained in the Resolution which, at the XXIInd General Assembly of the IAU, has prolonged the activity of the Group for another triennium. The basic motivations for the organization of this Workshop were: 1) to discuss the scientific, technical and managerial problems related to the possible set up of a surveillance system like the proposed Spaceguard System (see the "Spaceguard Survey Report", edited by D. Morrison, 1992, and the updated chapter contained in the book "Hazards due to Comets and Asteroids", edited by T. Gehrels, 1994); and 2) to put this discussion in an international context.

The five days of the Workshop were full of discussions on several topics, from the identification of observational sites, to the role of space activities, to the study of software and communication problems. The last day of the Workshop was devoted to a general discussion, during which it was proposed, and unanimously accepted, that a "Spaceguard Foundation" be established to provide an association among professionals and non-professionals aimed at sustaining and promoting NEO studies all around the world.

\subsection{THE SPACEGUARD FOUNDATION}

Following the decision taken at the Vulcano Workshop, The Spaceguard Foundation has been set-up, at the moment as an Italian association, on March 26, 1996. Thirty eight scientists, all of them belonging to the WGNEO, have acted as founders and are now Sustaining Members of the Foundation. According to the by-laws, the Foundation shall pursue the following objectives:

a) to promote and co-ordinate activities for the discovery, pursuit (follow-up) and orbital calculation of the NEO at an international level;

b) to promote study activities - at theoretical, observational and experimental levels - of the physicalmineralogical characteristics of the minor bodies of the solar system, with particular attention to the NEO;

c) to promote and co-ordinate a ground network (the "Spaceguard System"), backed up by a possible satellite network, for the discovery observations and for astrometric and physical follow-up.

The Board of Directors of The Foundation are: A. Carusi (President), D. I. Steel (Vice-President), S. Isobe, B. G. Marsden, K. Muinonen, E. M. Shoemaker. The Secretary is M. Carpino. All those interested in receiving information about the activity of the Foundation and on possible membership application should contact the Board at the address:

spaceguard@saturn.ias.fra.cnr.it

\subsection{THE RESOLUTION OF THE COUNCIL OF EUROPE}

While the Vulcano Workshop was being organized, the WGNEO was involved in the discussion and elaboration of a motion presented to the Committee on Science and Technology of the Council of Europe. After considerable discussion, and after a presentation given by A. Carusi at the Committee in Geneva, on March 20, 1996 the Parliamentary Assembly of the Council passed a Resolution (Res. 1080, 1996) which, recognizing the relevance of the threat posed by NEOs to humankind, and the necessity to deepen the studies in this field, concluded by stating:

" The Assembly invites governments of member states, those states enjoying observer status and the European Space Agency (ESA) to urge the setting-up and development of the above-mentioned Spaceguard Foundation and to give the necessary support to an international programme..." 


\subsection{THE FUTURE OF THE WGNEO}

After the setting-up of The Spaceguard Foundation it will be necessary to revise the status and membership of the WGNEO. Most of the members feel that it is still necessary to have an IAU Working Group on the subject, working in strict connection with the Foundation and in a form to be decided. The future activity of the WGNEO would consist mainly in maintaining a link to the Foundation and its affiliates, in reporting to the IAU about the developments and achievements of the Foundation, and in providing inputs and suggestions to its activity. 